\title{
Podocyte Injury Promotes Progressive Nephropathy in Zucker Diabetic Fatty Rats
}

\author{
Sachi Hoshi, Yujing Shu, Fusayo Yoshida, Tomoko Inagaki, Jiro Sonoda, \\ Teruo Watanabe, Ken-ichi Nomoto, and Michio Nagata
}

Department of Pathology, Institute of Basic Medical Sciences (SH, YS, TW) and Clinical Medicine (MN), University of Tsukuba; Department of Cardiovascular Research, Eisai Research Laboratories (FY, JS, K-IN), Tsukuba; and Department of Pathology, Showa University (TI), Tokyo, Japan

\begin{abstract}
SUMMARY: The zucker diabetic fatty (ZDF-fa/fa) rat is one of the attractive models for type II diabetes based on impaired glucose tolerance caused by the inherited insulin-resistance gene fa. Characterization of nephropathy in this model may provide useful insights into the mechanism of the progression of diabetic nephropathy. The present study analyzed the pathophysiology of diabetes and nephropathy, including the process of glomerulosclerosis in this model by biochemical and morphometric analyses. In addition, we conducted studies in podocytes in culture to examine the direct effects of high glucose on podocytes. ZDF-fa/fa rats showed overt diabetes despite hyperinsulinemia as early as 3 months of age. Blood glucose levels increased further with a considerable decrease of insulin levels at 5 months. Glomerular filtration rate (GFR) was significantly elevated until 3 months, but fell to the level seen in lean rats by 7 months. Proteinuria started to rise during the period of increased GFR, and increased further after GFR had fallen to within the normal range. Renal fibronectin, collagen iv, and vascular endothelial growth factor mRNA levels were increased at 7 months. Glomerulosclerosis commenced as early as 5 months of age, and was associated with glomerular hypertrophy and mild mesangial expansion with evidence of accentuated podocyte injury, as revealed by increased expression of desmin. Electron microscopy suggested that degeneration of podocytes and the development of tuft adhesions were responsible for the glomerular sclerosis in this model. In addition, glomeruli from the diabetic rats showed up-regulation of the cyclin kinase inhibitors, p21 and p27. Further studies suggested that the increase in p27 expression was predominantly caused by podocytes, because predominant immunolocalization of p27 in podocytes in diabetic rats and high glucose medium induced cell hypertrophy accompanied by p27 up-regulation in differentiated podocyte cell lines. In conclusion, progressive diabetic nephropathy in ZDF-fa/fa rats is associated with evidence of podocyte injury. High concentrations of ambient glucose induced podocyte hypertrophy and stress in vitro, suggesting that the podocyte is a likely target of the diabetic milieu. (Lab Invest 2002, 82:25-35).
\end{abstract}

$T$ he incidence of non-insulin-dependent diabetes mellitus (type II diabetes) is rapidly increasing world wide, particularly in Western countries (Ritz and Stefanski, 1996). Approximately $35 \%$ of patients needing renal replacement therapy have nephropathy associated with type II diabetes (Ritz and Stefanski, 1996). The rapid increase in the number of dialysis patients with diabetic nephropathy contributes importantly to rising medical costs, as well as adversely affecting the quality of life of such patients.

A dozen putative models of type II diabetes have been established, including ob/ob mice (Herberg and Coleman, 1977), db/db mice (Hummel et al, 1996), KK mice (Nakamura and Yamada, 1967), Goto-Kakizaki rats (Goto et al, 1976), obese zucker fa/fa rats (Godbole and York, 1978), and Otsuka Long Evans Tokushima fatty (OLETF) rats (Kawano et al, 1972). Most of these models

\section{Received September 4, 2001}

This work was supported by a research grant from of Tsukuba University and Health Sciences Research Grants of Japan (Research on Specific Diseases of Nephro-urological Study Group).

Address reprint requests to: Dr. Michio Nagata, Department of Pathology, Institute of Clinical Medicine, University of Tsukuba, Tsukuba, Ibaraki, 305-8575, Japan. E-mail: nagatam@md.tsukuba.ac.jp have abnormalities of single or multiple genes related to obesity, abnormal glucose tolerance, and/or insulin resistance leading to high blood glucose levels (Fiedorek, 1996). Although such models are useful in studying the early pathophysiology of type II diabetes, they are inadequate for examining progressive diabetic nephropathy with glomerulosclerosis. Indeed, OLETF rats showed persistent insulin resistance with glomerulosclerosis (Nakamura et al, 1997; Yagi et al, 1976), whereas renal injury in these models appears in older rats. Furthermore, the question remains whether early pathophysiologic changes are directly linked to glomerulosclerosis in rodent models of diabetes.

Obese zucker fa/fa rats have an autosomal recessive mutation in the fa gene that encodes the leptin receptor and show hyperphagia, obesity, and hyperlipidemia, but only mild elevation of blood glucose levels (Godbole and York, 1978). By crossbreeding obese zucker fa/fa rats with Wistar Kyoto rats, which are insulin-resistant and less tolerant to glucose, the zucker diabetic fatty (ZDF-fa/fa) rat was established (Ikeda et al, 1976). Overt diabetes was found from an early stage in this model, despite compensatory hypersecretion of insulin, indicating insulin resistance. Consequently, exhaustion of insulin secretion with 
impaired glucose tolerance promotes overt diabetes as early as 8 weeks of age. This mimics the pathophysiologic profile of human type II diabetes. ZDFfa/fa rats spontaneously develop diabetic nephropathy characterized by heavy proteinuria with focal segmental glomerulosclerosis as early as 16 weeks of age (Vora et al, 1996). However, the mechanism of progressive nephropathy in this model is unknown.

The present study was performed with the ZDF-fa/fa rats to investigate the pathophysiologic profile as a diabetic model, as well as the pathogenesis of glomerulosclerosis in this model. In addition, we tested the direct action of high glucose on podocytes using a differentiated mouse podocyte cell line. Our results suggested that progressive glomerulosclerosis in ZDF-fa/fa rats is based on podocyte damage. The direct action of glucose on differentiated podocytes in vitro suggested that podocytes are a primary target for the diabetic milieu and that podocyte injury accelerates the progression of glomerulosclerosis.

\section{Results}

\section{Parameters}

Table 1 shows several parameters related to diabetic nephropathy in ZDF-fa/fa rats and lean rats 3, 5, 7, and 9 months old. ZDF-fa/fa rats had higher body weight than lean rats until 3 months, but they tended to be less heavy at 5 months of age and were significantly lighter at 7 months. Kidney weight was significantly higher in ZDF-fa/fa rats than lean rats at all time points examined. Despite the presence of hyperinsulinemia, blood glucose levels were significantly higher in ZDF$\mathrm{fa} / \mathrm{fa}$ rats than in lean rats as early as 3 months. After 5 months, the elevation in the blood glucose levels was accelerated by a significant drop in plasma insulin levels. Proteinuria in ZDF-fa/fa rats was significantly higher at 5 months and was 8-fold higher at 7 months than in lean rats. Glomerular filtration rate (GFR) was significantly elevated in ZDF-fa/fa rats compared with lean rats at 3 and 5 months (approximately 1.9- and 1.7-fold higher, respectively). Notably, GFR in ZDF$\mathrm{fa} / \mathrm{fa}$ rats returned to a level similar to that in lean rats at 7 months. There was no significant difference in mean arterial pressure (MAP) between diabetic and lean rats at all time points examined.

\section{Renal Histology}

Glomerulosclerosis occurred as early as 5 months of age and tended to increase with age in the diabetic animals, whereas it was only rarely seen in the lean rats. Morphometry revealed that the incidence of sclerosis was significantly elevated in the diabetic rats with increased glomerular volume (GV). In addition, desmin score was significantly higher in the diabetic rats compared with the lean animals as early as 5 months and continued to increase thereafter (Fig. 1, A to C). We found good correlation between GV versus percentage glomerulosclerosis ( $p<0.05$ ), GV versus desmin score $(p<0.01)$, and percentage glomerulosclerosis versus desmin score $(p<0.01$ ) in ZDF-fa/fa rats (Fig. 1, D to F).

The stepwise development of glomerulosclerosis in ZDF-fa/fa rats is shown in Figure 2 as revealed by high-resolution light microscopy. The earliest glomerular changes in ZDF-fa/fa rats were glomerular enlargement with mild mesangial widening found as early as 5 months. Podocytes showed droplet inclusions and pseudocysts to some extent. Segmental sclerosis was built on tuft adhesion to Bowman's capsule on a background of an increased incidence and severity of podocyte injury as revealed by desmin expression. Mesangial expansion was mild at best and insufficient for mesangial sclerosis or mesangial nodules as typically found in human diabetic nephropathy. Synechiae extended to segmental sclerosis and glomerular obsolescence with hyaline deposition. Electron microscopy revealed frequent degeneration and detachment of podocytes in nonsclerotic glomeruli. In the area of synechiae, the glomerular basement membrane (GBM) showed a covering by parietal epithelial cells. In the sclerotic segment, the glomerular tuft was collapsed with extensive denudation of GBM (Fig. 3). No electron-dense deposits were observed. Interstitial fibrosis and tubular atrophy with inflammatory infiltration tended to be spatially associated with glomerulosclerosis. Immunostaining for desmin was conspicuous in the podocytes in nonsclerotic glomeruli in ZDF-fa/fa rats (Fig. 4). Hydronephrosis or necrotizing granulomatous lesions were absent in all animals.

Table 1. Pathophysiological Data of ZDF-fa/fa and Lean Rats

\begin{tabular}{|c|c|c|c|c|c|c|c|c|}
\hline & & $\begin{array}{c}\text { Body weight } \\
\mathrm{g}\end{array}$ & $\begin{array}{c}\text { Kidney weight } \\
\text { g }\end{array}$ & $\begin{array}{c}\text { Blood glucose } \\
\text { mg/dl }\end{array}$ & $\begin{array}{c}\text { Plasma insulin } \\
\mathrm{ng} / \mathrm{ml}\end{array}$ & $\begin{array}{l}\text { MAP } \\
\mathrm{mmHg}\end{array}$ & $\begin{array}{l}\text { GFR } \\
\mathrm{ml} / \mathrm{min}\end{array}$ & $\begin{array}{l}\text { Proteinuria } \\
\text { mg/day }\end{array}$ \\
\hline \multirow[t]{2}{*}{$3 \mathrm{mo}$} & Lean & $256 \pm 18$ & $2.17 \pm 0.11$ & $123 \pm 6$ & $1.16 \pm 0.17$ & $111 \pm 2$ & $0.37 \pm 0.10$ & $7.3 \pm 2.1$ \\
\hline & $f a / f a$ & $337 \pm 19^{* *}$ & $3.32 \pm 0.11^{\star *}$ & $337 \pm 58^{\star}$ & $6.47 \pm 1.88^{*}$ & $125 \pm 9$ & $0.72 \pm 0.08^{*}$ & $30.6 \pm 12.5$ \\
\hline \multirow[t]{2}{*}{$5 \mathrm{mo}$} & Lean & $424 \pm 11$ & $2.58 \pm 0.04$ & $140 \pm 5$ & $2.42 \pm 0.45$ & $130 \pm 9$ & $2.30 \pm 0.18$ & $7.2 \pm 1.6$ \\
\hline & $f a / f a$ & $440 \pm 8$ & $3.58 \pm 0.15^{\star \star}$ & $512 \pm 62^{\star \star}$ & $1.87 \pm 0.26$ & $118 \pm 7$ & $3.80 \pm 0.57^{\star}$ & $68.7 \pm 15.3^{\star \star}$ \\
\hline \multirow[t]{2}{*}{$7 \mathrm{mo}$} & Lean & $461 \pm 8$ & $2.60 \pm 0.11$ & $134 \pm 3$ & $2.45 \pm 0.28$ & $123 \pm 4$ & $1.90 \pm 0.20$ & $32.8 \pm 10.0$ \\
\hline & $\mathrm{fa} / \mathrm{fa}$ & $425 \pm 10^{*}$ & $4.10 \pm 0.15^{\star \star}$ & $576 \pm 57^{* *}$ & $1.25 \pm 0.16^{\star \star}$ & $131 \pm 7$ & $1.80 \pm 0.12$ & $258.3 \pm 114.9^{\star *}$ \\
\hline \multirow[t]{2}{*}{$9 \mathrm{mo}$} & Lean & $480 \pm 5$ & $2.62 \pm 0.15$ & $196 \pm 22$ & $3.77 \pm 0.51$ & $126 \pm 4$ & $1.75 \pm 0.50$ & $14.0 \pm 3.2$ \\
\hline & $f a / f a$ & $412 \pm 9^{\star *}$ & $4.23 \pm 0.20$ ** & $567 \pm 28^{* *}$ & $0.99 \pm 0.17^{\star *}$ & $140 \pm 8$ & $1.85 \pm 0.25$ & $286.0 \pm 59.0^{* *}$ \\
\hline
\end{tabular}

Values are expressed as means $\pm \mathrm{SEM} ; n=6$ rats per group.

MAP, mean arterial pressure; GFR, glomerular filtration rate.

${ }^{*} p<0.05,{ }^{*} p<0.01$ versus ZDF-lean rats of the same age. 
A

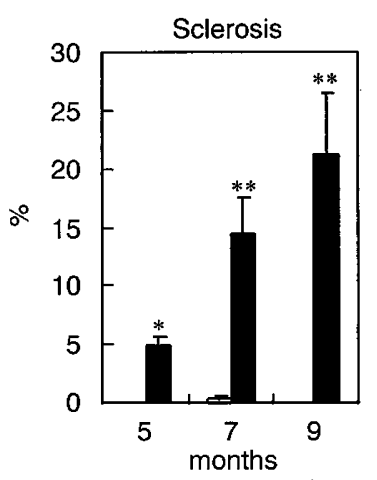

D

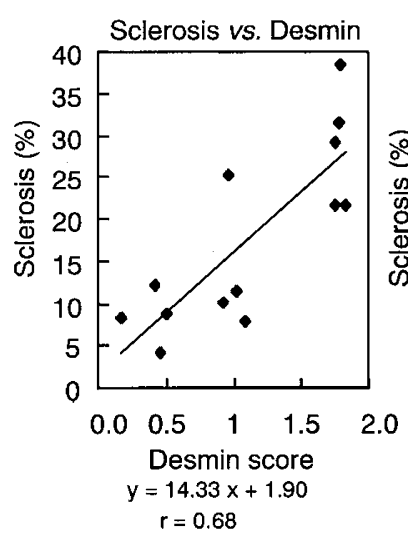

B

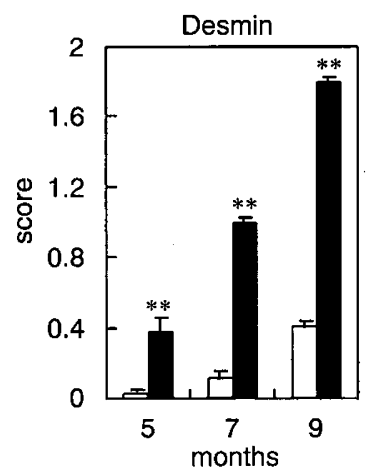

$\mathrm{E}$

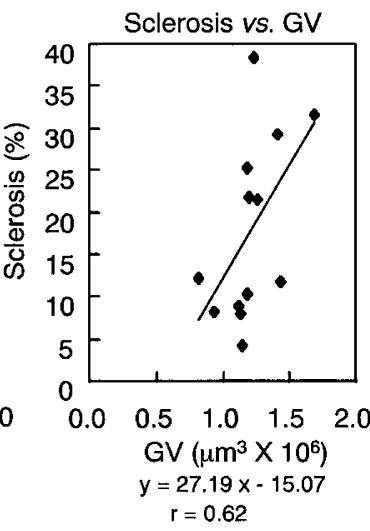

C

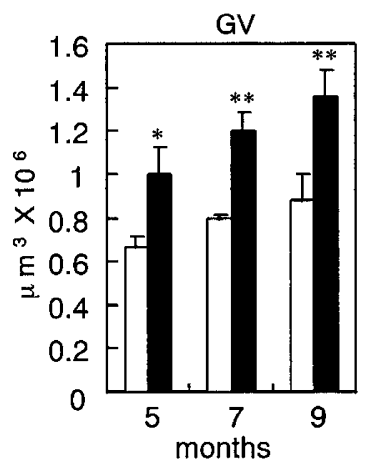

$\mathrm{F}$

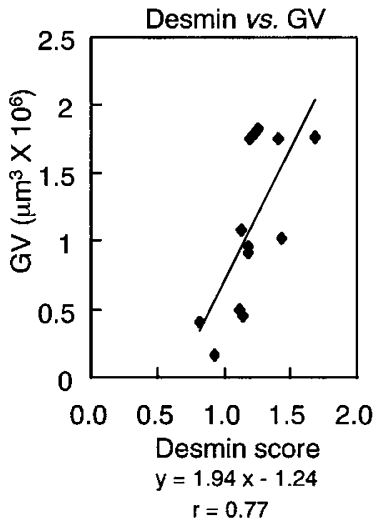

Figure 1.

Morphometric analysis of glomeruli in ZDF fa/fa $(\square)$ and lean rats ( $\square$ ). Incidence of glomerulosclerosis (A), desmin score (B), and glomerular volume (GV) (C) was significantly higher in ZDF-fa/fa rats compared with the lean controls as early as 5 months and increased further thereafter. ${ }^{\star \star} p<0.01,{ }^{\star} p<0.05$. The lower panels show the correlation between the percentage glomerulosclerosis and glomerular volume (D), percentage glomerulosclerosis and desmin score (E), and glomerular volume and desmin score $(\mathrm{F})$.

\section{Expression of Fibronectin, Type iv Collagen, and Vascular Endothelial Growth Factor (VEGF) mRNA}

Figure 5 shows a Northern blot of the kidney cortex mRNA for fibronectin, type iv collagen, and VEGF in ZDF-fa/fa and lean rats. In ZDF-fa/fa rats, mRNA levels of fibronectin, type iv collagen, and VEGF tended to increase with age. Collagen iv and VEGF mRNA levels of ZDF-fa/fa rats at 5 months were significantly higher than those of lean rats. At 7 months of age, levels of all these mRNA in ZDF-fa/fa rats were significantly increased compared with lean rats.

\section{Glomerular Expression of Cyclin-Dependent Kinase Inhibitors}

Western blot analysis revealed that expression of p21 and p27 was significantly increased in ZDF-fa/fa rats compared with lean rats $(1.58 \pm 0.23, p<0.05$, and $1.78 \pm 0.13, p<0.01$, respectively; Fig. 6 ). The levels of p57 were unchanged $(1.04 \pm 0.06$ levels in lean rats). In addition, immunostaining of p27 in the glomeruli of diabetic rats showed predominant localization in podocytes (data not shown).

\section{Effects of High Glucose on Immortalized Mouse Podocyte Cell Line under Nonpermissive Condition}

Immortalized mouse podocytes under permissive conditions with normal glucose (NG) revealed a cobblestone appearance (Fig. 7A, left panel). Under nonpermissive conditions of $37^{\circ} \mathrm{C}$ without IFN- $\gamma$, the cells began to show an arborized shape (Fig. 7A, center panel). Podocytes under nonpermissive conditions with high glucose (HG) exhibited cell hypertrophy (Fig. 7A, right panel). ${ }^{3} \mathrm{H}$-thymidine incorporation was not different among the three treatment groups. In contrast, ${ }^{3} \mathrm{H}$-leucin incorporation under high glucose conditions was significantly elevated compared with cells treated with NG or NG with mannitol (MN) (Fig. 7B). Western blotting analysis showed that p27 levels were significantly higher in podocytes with high glucose than in normal glucose, whereas p21 and p57 levels did not differ among the three groups. Quantitative analysis by densitometry of Western blots gave the following results: p21: HG, $1.05 \pm 0.07$ of NG; MN, $0.93 \pm$ 0.15 of NG; p27: HG, $1.52 \pm 0.12$ of NG $(p<0.05)$; $\mathrm{MN}, 1.32 \pm 0.14$ of NG; p57: HG, $0.99 \pm 0.07$ of NG; $\mathrm{MN}, 1.05 \pm 0.08$ of NG. 


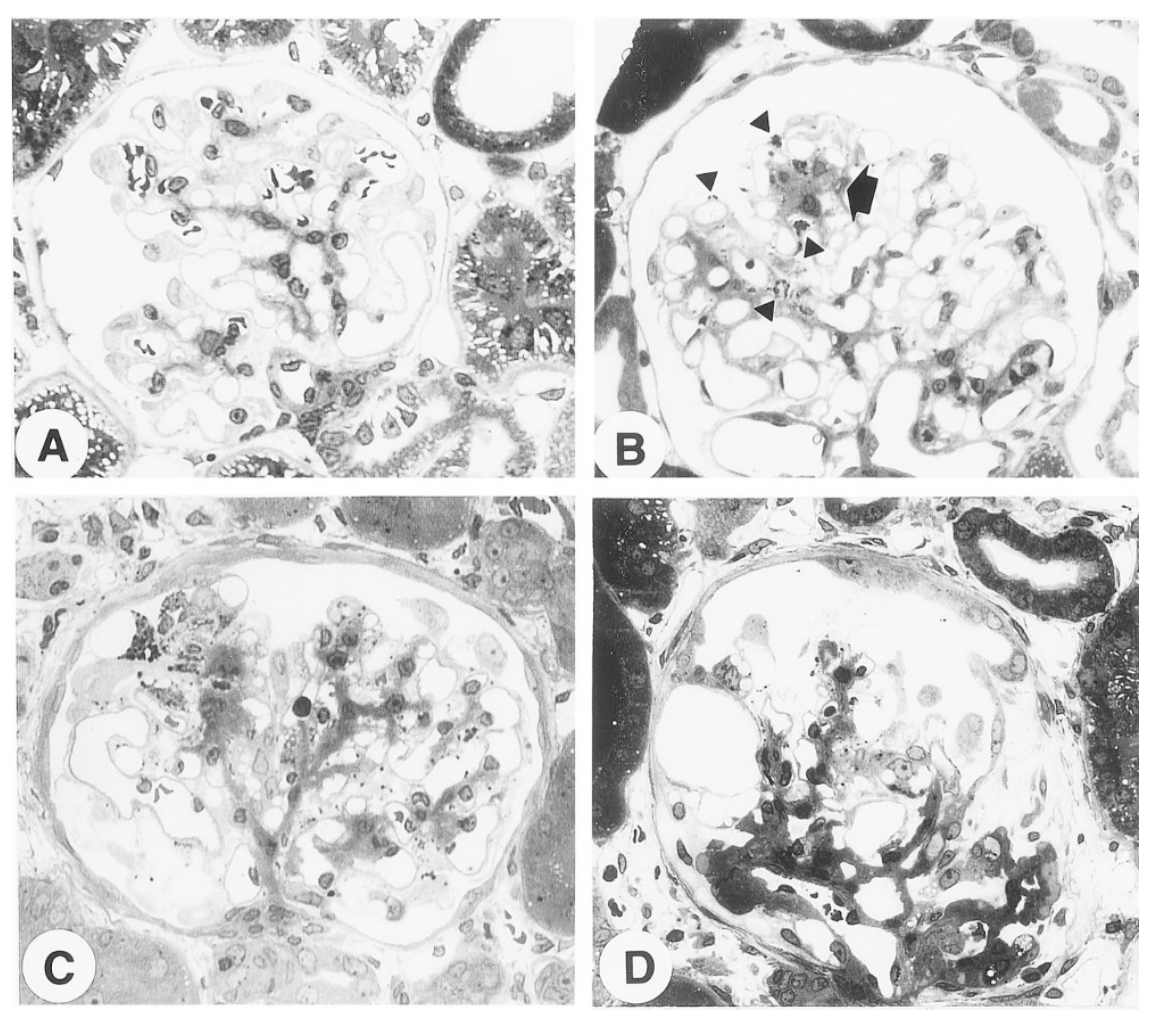

Figure 2.

High-resolution microscopic features of the glomerular profile in ZDF-fa/fa rats. A, Glomeruli from 5-month-old lean rats showing neither apparent glomerular enlargement nor mesangial expansion. B, Glomerulus at 5 months in a ZDF-fa/fa rat showing some hypertrophy of glomerular tuft with segmental mesangial accentuation (arrow) and scattered cytoplasmic deposition in podocytes (arrowheads). C, Glomerulus from a 7-month-old ZDF-fa/fa rat. Mild mesangial thickening with synechiae can be seen. Note conspicuous droplets and vacuolation in podocytes. No nodular lesions were seen. D, Glomerulus from a ZDF-fa/fa rat at 7 months. Note segmental sclerosis with capillary collapse near the vascular pole. Original magnification, A-D, $\times 400$.

\section{Discussion}

Reflecting the genetic abnormality of the fa gene and glucose intolerance, ZDF-fa/fa rats showed high blood glucose levels despite considerable hyperinsulinemia at an early stage of disease. At a later stage, plasma insulin levels dropped significantly and blood glucose levels were further elevated. The physiologic profile of insulin resistance and glucose intolerance is similar to human type II diabetes. In addition, this model showed an earlier occurrence of glomerulosclerosis than any other model of diabetes. However, the mechanism of glomerulosclerosis in this model was not known.

Diabetic nephropathy in general is characterized by renal hypertrophy, hyperfiltration, proteinuria, and progressive glomerulosclerosis. In the present study, ZDF-fa/fa rats showed significant renal hypertrophy compared with lean rats as early as 3 months of age. The levels of fibronectin, collagen type IV, and VEGF mRNA in the kidney were elevated in ZDF-fa/fa rats, as previously reported in other models of diabetes (Cohen et al, 1995; Tsuchida et al, 1999). In addition, ZDF-fa/fa rats showed a transient increase in GFR level in the early to middle stages of the disease, with glomerular hypertrophy indicating the presence of hyperfiltration. Onset of proteinuria and glomerulosclerosis was associated with hyperfiltration, but both proteinuria and sclerosis continued to increase after apparent normalization of GFR. This indicated that hyperfiltration in the early stage of disease contributes to the evolution of nephropathy and progressive glomerulosclerosis in the late stage may not be due to hyperfiltration per se, or that continuing hyperfiltration compensates poorly for progressive glomerulosclerosis. Notably, blood pressure was not elevated in diabetic animals compared with the lean controls throughout the experiment. In addition, body weight was transiently higher in the diabetic rats at 3 months and dropped to the level of the lean rats before glomerulosclerosis became advanced. Thus, the diabetic milieu, but not hypertension or obesity, may be a predominant factor in promoting progressive nephropathy in this diabetic model.

The mechanism responsible for the glomerulosclerosis seen in this model has not been determined. Previously, Vora et al (1996) reported glomerulosclerosis in ZDF/Drf-fa rats, the same strain as used in the present study. Although Vora and colleagues did not focus on the mechanism of glomerulosclerosis, they suggested that hydronephrosis or necrotizing lesions were involved in the progressive glomerulosclerosis in these animals. However, our animals did not show hydronephrosis or necrotizing lesions. In addition, our diabetic rats showed renal and glomerular hypertrophy with hyperfiltration, indicating the occurrence of diabetic nephropathy. Glomerulosclerosis in this model was established on tuft adhesion to Bowman's 

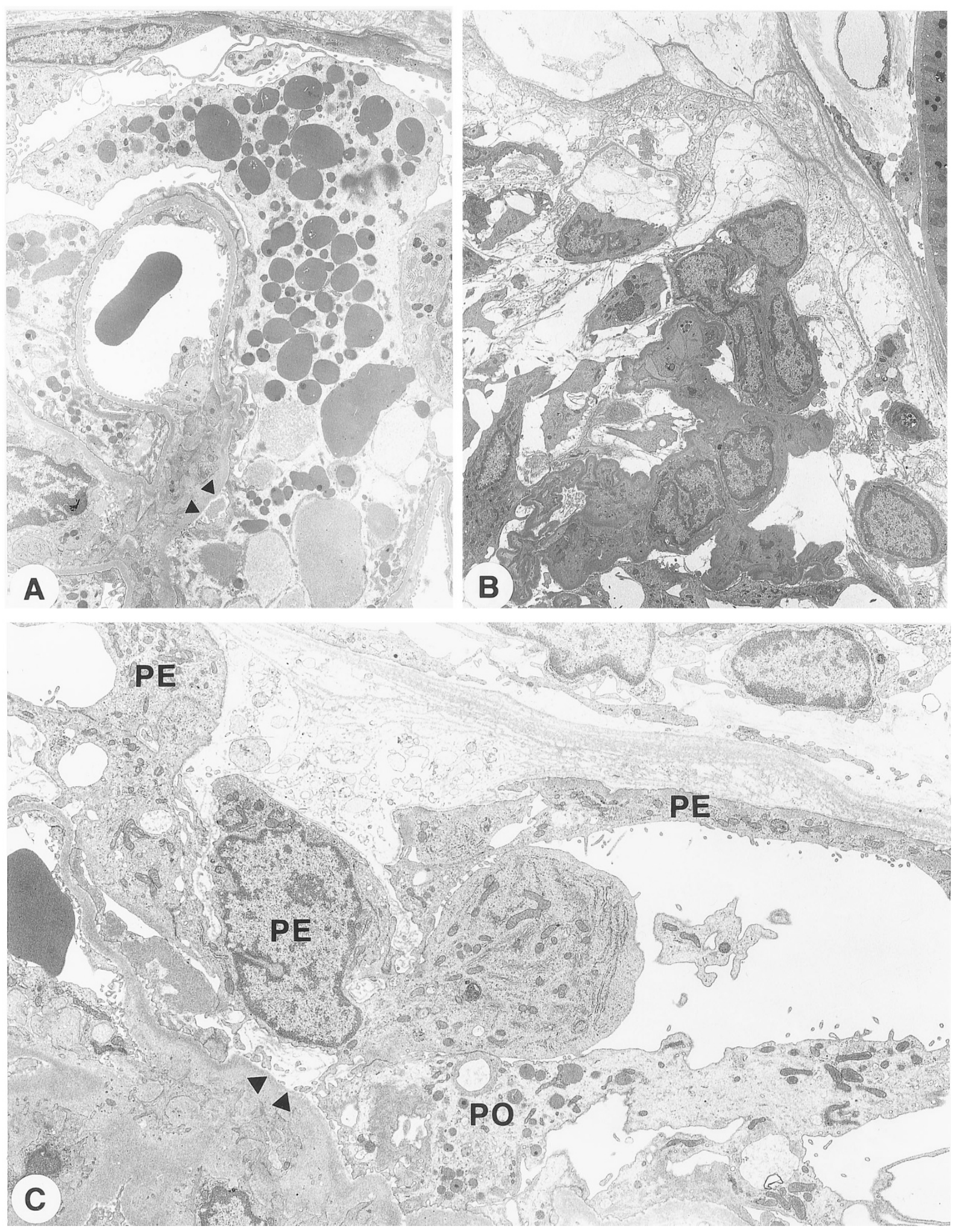

Figure 3.

Electron microscopic features of ZDF-fa/fa rats at 5 months. A, Hypertrophy in podocytes was associated with cytoplasmic droplets and vacuolation. Diffuse effacement of foot processes with actin accumulation can be seen. Note the focus of detachment (arrowheads). Mesangium and endothelial cells show no apparent abnormalities. B, Area of segmental sclerosis. Extensive denudation of glomerular basement membrane on the collapsed tuft can be seen. Note tuft adhesion with thickening of the basement membrane in Bowman's capsule. C, A focus of adhesion. Reactive parietal epithelial cells $(P E)$ attached to the site of focal detachment (arrowheads) of podocytes (PO). Original magnification, A, $\times 4000$; B, $\times 2500$; C, $\times 5500$. 

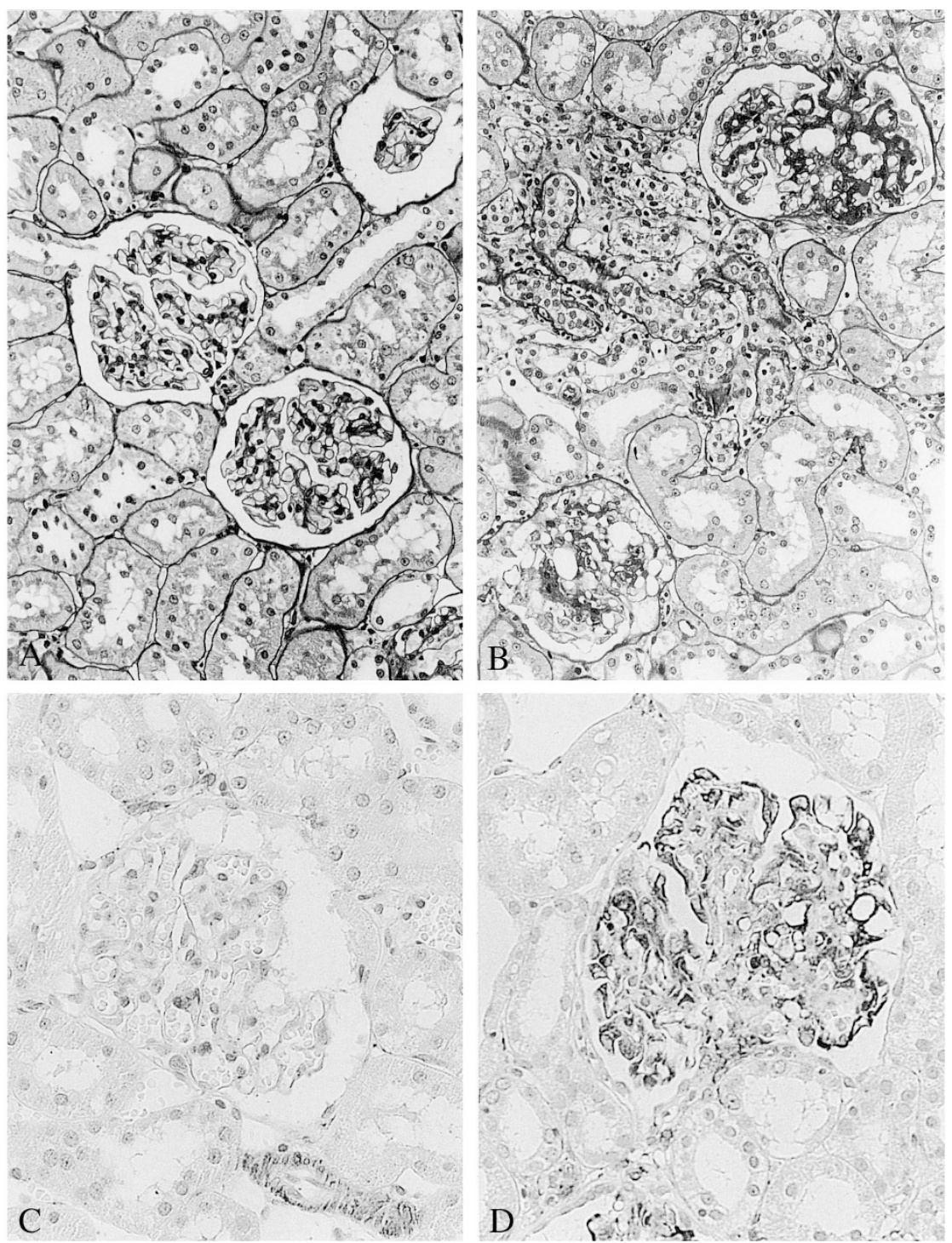

Figure 4.

Renal histology and desmin expression in lean and ZDF fa/fa rats. Representative renal histology (HE) and glomerular desmin expression in lean rats (A), ZDF-fa/fa rats (fa/fa), (B) at 5 months. ZDF-fa/fa rats showed glomerulosclerosis with focal tubular atrophy, interstitial fibrosis and inflammatory infiltrates accompanied by segmental glomerulosclerosis. Desmin was strongly expressed in podocytes with some vacuolation in ZDF-fa/fa rats at 5 months (D), whereas staining was minimal in the lean rats (C). Original magnification, $A$ and $B, \times 200 ; C$ and $D, \times 350$.

capsule and the degree of mesangial widening was generally mild and insufficient to cause development of intracapillary mesangial sclerosis or Kimmelstiel Wilson nodules, which are the typical feature of glomerulosclerosis in human diabetes. In contrast, desmin immunostaining, a marker of podocyte injury (Yaoita et al, 1990), was significantly elevated with proteinuria and glomerular hypertrophy. Electron microscopy indicated that the early glomerular changes in this diabetic model were podocyte injury that led to detachment of podocytes and tuft adhesion. These features are the characteristics of recently defined podocyte-oriented common pathway to glomerulosclerosis (Kriz et al, 1994). Notably, glomeruli from the diabetic animals showed up-regulation of cyclindependent kinase inhibitor (CKI), p21, and p27 by Western blot. This finding was consistent with recent observations showing up-regulation of $\mathrm{CKI}$ in diabetic mice (Kuan et al, 1998; Wolf et al, 1998). The p27 are predominantly expressed in podocytes within the glomeruli to stabilize the cell cycle of these terminally differentiated cells (Nagata et al, 1998) and immunostaining for p27 in diabetic animals showed predominant expression in podocytes. Up-regulation of p27 has been found in the model of experimental membranous glomerulonephritis, a model of podocyte injury (Shankland et al, 1997). We found strong immunostaining of p27 in the remnant glomeruli of crescentic glomerulonephritis (Nitta et al, 1999). In addition, p27 knockout mice developed more severe renal damage (Ophascharoensuk et al, 1998). Finally, re-entry of podocytes into the cell cycle has been found to result in heavy proteinuria and glomerulosclerosis (Kriz et al, 1995). From these observations, it is 
A

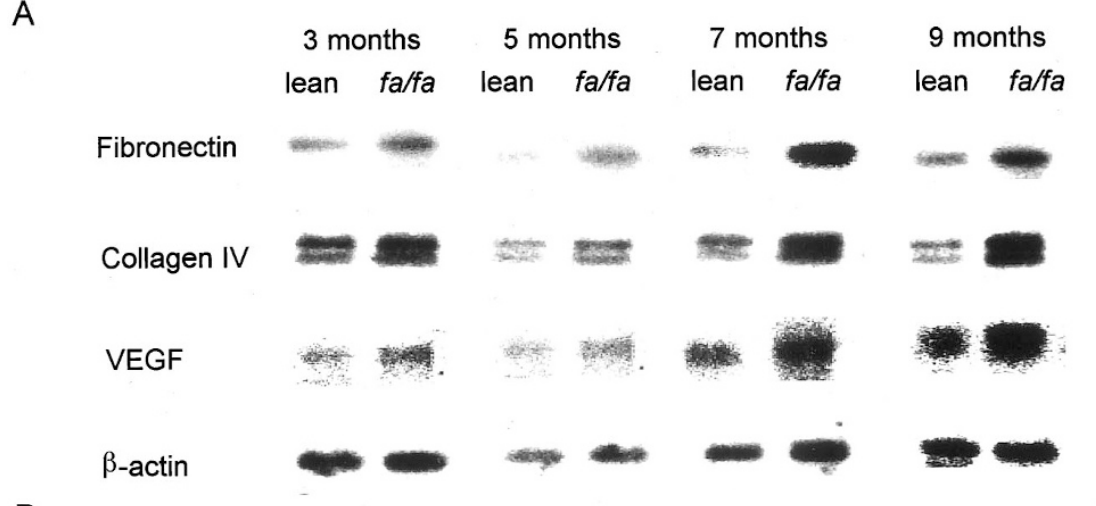

B
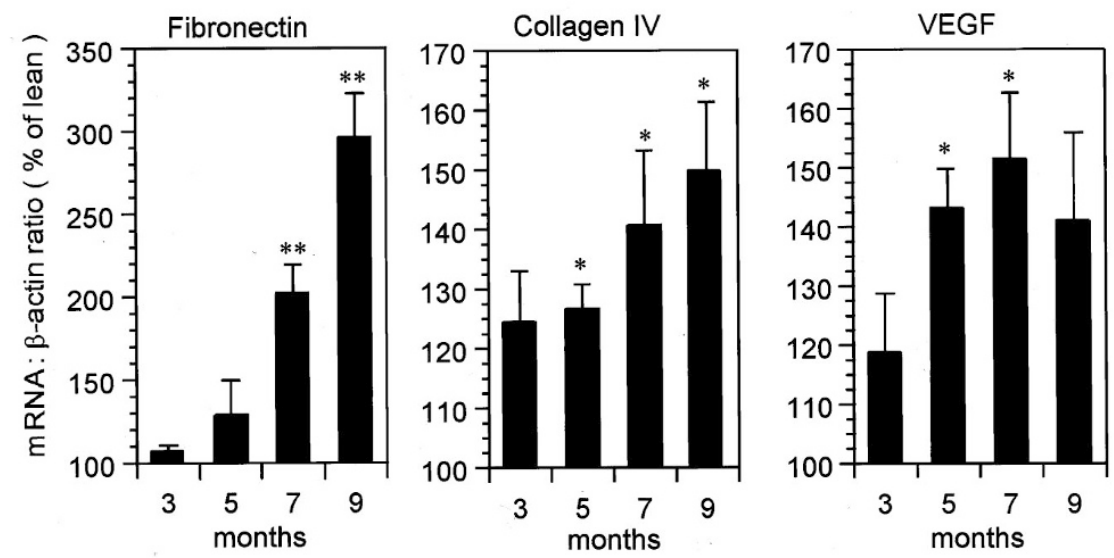

Figure 5.

Northern blotting analysis of fibronectin, collagen type iv, and VEGF mRNA at 3, 5, 7, and 9 months in kidneys from ZDF-fa/fa (fa/fa) and lean rats (lean). Equivalent amounts of total RNA ( $15 \mu \mathrm{g} / \mathrm{lane}$ ) of each sample were analyzed by Northern blot hybridization using three cDNA probes or a $\beta$-actin cDNA probe. A, Results of one representative experiment are shown. B, The intensity of each band was calculated with an image analyzer in relation to the $\beta$-actin mRNA level. Results represent means \pm SEM of three independent experiments and the ratios are expressed as percentages of the value for age-matched lean rats. ${ }^{\star} p<0.05,{ }^{\star \star} p<0.01$ versus lean.

tempting to speculate that elevation of p27 in the glomeruli in the diabetic rats in our study is a cellprotective phenomenon in podocytes, reflecting the presence of injury or stress in vivo. Taken together, the present study suggests that ZDF-fa/fa rats demonstrate progressive glomerulosclerosis associated with type II diabetic phenotype via a mechanism based on podocyte injury.

Mesangial nodular lesions, characteristic features of human diabetic nephropathy, were not seen in ZDF$\mathrm{fa} / \mathrm{fa}$ rats. In this context, it may be noteworthy that the early glomerular changes in zucker fa/fa rats and Goto Kakizaki rats with type II diabetes were characterized by podocyte injury without apparent mesangial expansion (Coimbra et al, 2000; Phillips et al, 1999). We suggest that rats are not susceptible to mesangial nodular lesions in overt diabetes, and that podocyte damage may be a distinct pathology of diabetic milieu in rodents.

The importance of podocyte injury for progressive diabetic nephropathy in type II diabetes has also been noted in humans. Recent reports suggested that podocyte loss was important for the progression of glomerulosclerosis in Pima Indians with type II diabetes (Nelson et al, 1997; Pagtalumn et al, 1997). However, the morphometric data of these reports did not provide evidence regarding the mechanism of podocyte loss, for example, whether podocytes loss was the result of degeneration and detachment due to the direct action of high glucose on the podocyte. Nakamura et al (2000) reported that podocytes in the urine are correlated with disease activ-

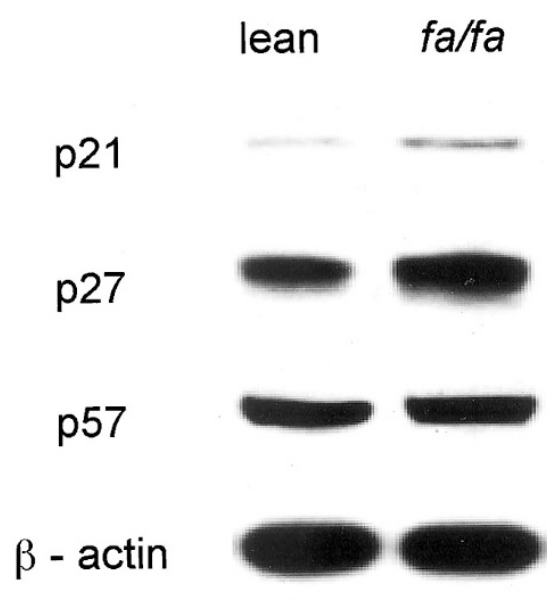

Figure 6.

Expression of p21, p27, and p57 at 5 months in glomeruli from ZDF-fa/fa (fa/fa) and lean rats. Equivalent amounts ( $40 \mu \mathrm{g} / \mathrm{lane})$ of each sample were analyzed by Western blotting analysis. The results were derived from three rats from each group. Western blotting was independently performed five times. Representative data of one experiment are shown. Quantitative and statistical data are described in the text. 
A

$33^{\circ} \mathrm{C} \quad \mathrm{IFN}_{\gamma}(+) \quad \mathrm{NG}$

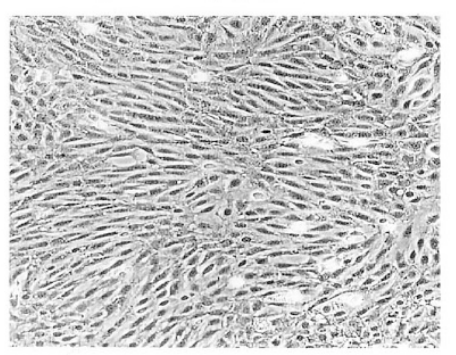

$37^{\circ} \mathrm{C} \quad$ IFN $\gamma(-) \quad N G$

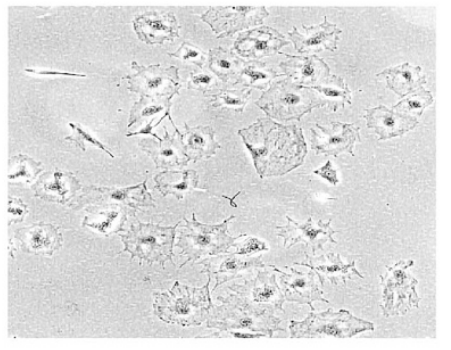

$37^{\circ} \mathrm{C} \quad$ IFN $\gamma(-)$ HG

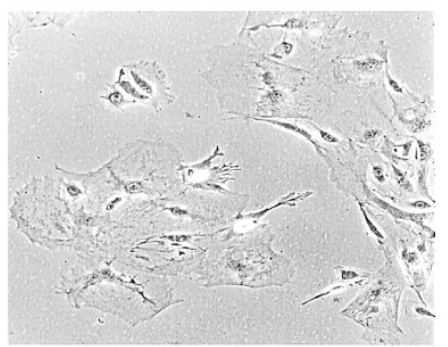

B

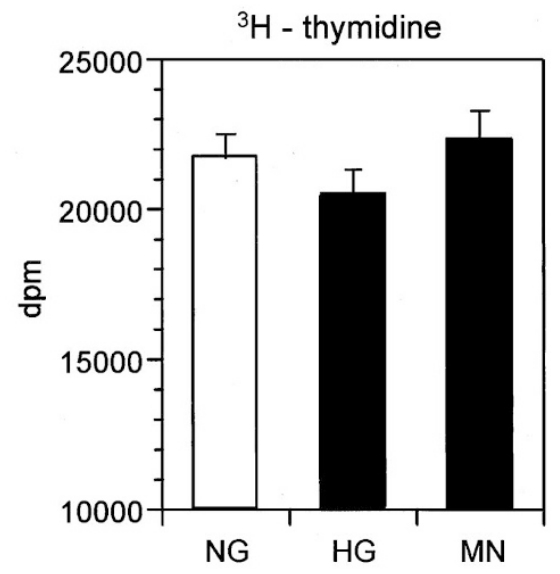

C

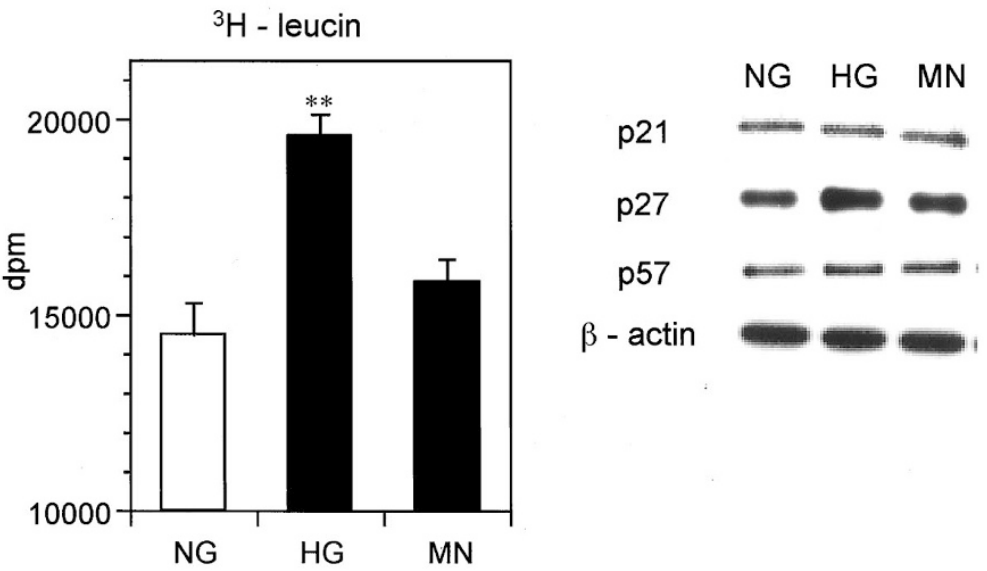

Figure 7.

Culture of immortalized podocyte cell line. A, Podocyte cell line grown at $33^{\circ} \mathrm{C}$ with $100 \mathrm{U} / \mathrm{ml} \mathrm{IFN-} \gamma$ (left panel). Differentiated arborized podocytes at $37^{\circ} \mathrm{C}$, no IFN- $\gamma$ under normal glucose conditions ( $5.5 \mathrm{~mm}$ glucose; middle panel) or high glucose conditions for 3 days ( $25 \mathrm{~mm}$ glucose; right panel). Original magnification, $\times 150$. $\mathrm{B},{ }^{3} \mathrm{H}$-thymidine incorporation (left) and ${ }^{3} \mathrm{H}$-leucin incorporation (right) into differentiated mouse podocyte cell line under normal glucose (NG), high glucose (HG), or normal glucose with mannitol $(M M)$. Results represent means \pm SEM of six independent cultures. ${ }^{\star \star} p<0.01$ versus NG. C, Expression of $p 21$, p27, p57, and $\beta$-actin. Equivalent amounts (10 $\mu \mathrm{g} / \mathrm{lane})$ of each sample were analyzed by Western blotting. Western blotting was independently performed five times. Representative data from one experiment are shown. Quantitative and statistical data are described in the text.

ity in diabetic nephropathy, suggesting that the diabetic milieu damages podocytes in humans. Many factors may be synergistically involved in podocyte injury in the diabetic condition. In fact, we found good correlation between glomerular volume and desmin score in ZDFfa/fa rats. This suggests that diabetes-induced glomerular hypertrophy is one of the factors promoting podocyte injury in this model, as previously shown in the remnant kidney model (Nagata and Kriz, 1992). In addition, the present study tested for the first time the direct effects of high glucose on cellular changes in podocytes in vitro. Using differentiated mouse podocytes cultured under nonpermissive conditions, we found that podocytes underwent cell hypertrophy, but not hyperplasia, with up-regulation of p27. Lack of p21 up-regulation under high glucose in the podocyte cell line was consistent with the previous findings that mesangial cells were the predominant cells expressing p21 in diabetic conditions in vitro and in vivo (Kuan et al, 1998). Because mannitol-treated cells were not hypertrophic and showed no elevation of p27 compared with normal glucose-treated cells, these cellular changes in cultured podocytes were likely to be due to the direct action of glucose on podocytes, rather than being due to high osmolality. This result supported the notion that p27 up-regulation in ZDF-fa/fa rats may be partly due to direct action of glucose on podocytes. Although the mechanism responsible for the up-regulation of p27 in podocytes induced by high glucose is unknown, upregulation of p27 in podocytes may reflect glucoseinduced cellular changes (stress or injury?) in podocytes. Further studies are needed to characterize the "stress" in podocytes in a high-glucose environment.

In conclusion, the present study characterized for the first time that podocyte damage is the underlying mechanism responsible for the promotion of progressive diabetic nephropathy in ZDF-fa/fa rats. In addition, the action of high glucose on podocytes in vitro suggested that glucose-induced podocyte stress is one of the factors promoting nephropathy in type II diabetes.

\section{Materials and Methods}

\section{Animals and Experimental Protocol}

Seven-week-old ZDF-fa/fa rats and ZDF-lean male rats (lean, as a control) were obtained from Genetic 
Models (Indianapolis, Indiana). All rats were kept in temperature- and humidity-controlled rooms and were fed standard chow ad libitum. Three groups of ZDF$\mathrm{fa} / \mathrm{fa}$ rats and three groups of lean (six in each) rats were studied at the ages of $3,5,7$, and 9 months. In each experimental period, body weight, proteinuria, blood glucose, and insulin levels were measured. On the next day, GFR was estimated by inulin clearance and animals were then anesthesized by Nembutal (Dainippon Seiyaku Co., Osaka, Japan) and killed to obtain kidney samples. Kidneys were fixed in formalin or $2.5 \%$ glutaraldehyde for histology or snap frozen and stored at $-80^{\circ} \mathrm{C}$ until extraction of total RNA and protein.

\section{Measurement of GFR and Blood Pressure}

GFR was determined by inulin clearance (Davidson and Sackner, 1963). Briefly, the rats were anesthetized with intraperitoneal injection of pentobarbital sodium $(30 \mathrm{mg} / \mathrm{kg})$. Catheters were then inserted into the left carotid artery for blood pressure monitoring and blood sampling, and into the urinary bladder for urine collection. Four percent of inulin in normal saline was infused with $0.03 \mathrm{ml}$ per minute for 50 minutes via suprajugular vein. Then, successive clearance studies in 20 minutes were performed twice. The concentrations of inulin in the plasma and urine were determined according to the anthrone reaction.

\section{Histopathology}

Formalin-fixed kidney slices were embedded in paraffin, sectioned, and stained with hematoxylin-eosin and periodic acid-Schiff. Mean glomerular volume in each animal was calculated from the value of mean planar area in all glomerular profiles in random sections with a computer using the method of Weibel as previously described (Nagata and Kriz, 1992). Approximately 200 glomeruli were included in each section. Incidence of glomerulosclerosis was determined by percentage of sclerotic glomeruli for all glomerular profiles in single sections ( $213 \pm 5$ glomerular profiles) in each rat. To estimate the extent of podocyte injury, desmin immunostaining with anti-desmin monoclonal antibody (DAKO, Gostrup, Denmark) was performed by the standard immunostaining procedure (Nagata et al, 1998; Yaoita et al, 1990). Desmin in each nonsclerotic glomerulus was scored from 0 to 3 based on the relative intensity of immunolabeling. The average value of the desmin score in 50 randomly selected nonsclerotic glomeruli in each animal was taken to represent the degree of podocyte injury. The profile of each animal was masked at histologic examination. Immunolabeling of p27 (Santa Cruz Biochemicals, Santa Cruz, California) was performed with the same protocol.

\section{RNA Isolation and Northern Blot Hybridization}

Total RNA was isolated from frozen kidney samples by acid guanidinium isothiocyanate extraction (Chomczynski and Sacchi, 1998). Aliquots of $15 \mu \mathrm{g}$ of total RNA were subjected to electrophoresis in $1.2 \%$ agarose/ formaldehyde gels at $80 \mathrm{~V}$ for 3 to 4 hours, and transferred for 12 to 16 hours to charged nylon membranes (zeta-probe blotting membrane). The cDNA probes for rat fibronectin, collagen type iv, and VEGF were synthesized by PCR using specific oligonucleotide primers and rat kidney $\mathrm{CDNA}$ as a template. The primer pairs were: for fibronectin, sense, $5^{\prime}$-ttt tga caa cgg gaa gca tta tca gat aa-3', and antisense, 5'-tga tca aaa cat ttc tca gct att gg-3'; for collagen type iv, sense, $5^{\prime}$-ctc tgg gga caa cat ccg-3', and antisense, 5'-tct tct cat gca cac ttg gc-3'; and for VEGF, sense, $5^{\prime}$-atg aac ttt ctg ctg tct tgg gtg cat tgg-3', antisense, 5'-tca ccg cct cgg ctt gtc aca-3'. These PCR products were subcloned with a TA cloning kit (Invitrogen, Carlsbad, California), and their nucleotide sequences were confirmed by comparison with data in NCBI. cDNA was excised by EcoRI and labeled with $\left[\alpha^{32} \mathrm{P}\right] \mathrm{dCTP}$ by extension of random hexamer primers (Amersham Pharmacia Biotech, Piscataway, New Jersey). Blots were washed twice $\left(0.1 \times \mathrm{SSC}, 65^{\circ} \mathrm{C}, 15\right.$ minutes) and hybridization signals were detected and quantified with the BAS2000 system (Fuji Film, Tokyo, Japan). All results were corrected using $\beta$-actin as the standard cDNA probe (CLONTECH, Palo Alto, California). For each sample, the experiment was repeated three times.

\section{Biochemical Analysis}

Urine was collected in metabolic cages for 24 hours. Proteinuria was measured with a protein assay kit (Bio-Rad, Tokyo) and bovine serum albumin was used as a standard. Insulin concentrations were measured using an enzyme-linked immunosorbent assay (EIA) kit for insulin (Morinaga, Yokohama, Japan). Blood glucose levels were measured with a $\beta$-glucose analyzer (Wako Chemical, Tokyo, Japan).

\section{Cell Culture}

The immortalized mouse podocyte clone was a generous gift from Dr. Mundel (Mundel et al, 1997) and was maintained in Dulbecco's modified Eagle's medium (DMEM, low glucose type; Invitrogen) supplemented with $10 \%$ FBS and $10 \mathrm{U} / \mathrm{ml}$ recombinant mouse IFN- $\gamma$ (Sigma, St. Louis, Missouri) at $33^{\circ} \mathrm{C}$. To induce differentiation, podocytes were resuspended on type I collagen-coated dishes (Iwaki Glass, Tokyo, Japan) at $37^{\circ} \mathrm{C}$ without IFN- $\gamma$ (nonpermissive condition). After 7 days under nonpermissive conditions, the majority of cells showed arborized shape and expressed synaptopodin (Mundel et al, 1997).

\section{${ }^{3} \mathrm{H}$-Thymidine and ${ }^{3} \mathrm{H}$-Leucin Incorporation}

Podocytes cultured under nonpermissive conditions for 4 days were used for the experiment. After most of the cells showed an arborized shape, they were starved in DMEM containing $0.2 \%$ BSA and $0.4 \%$ FCS for 48 hours. Then, the cells were incubated with NG (5.5 mM), HG (25 mM), or MN (19.5 mm), which served as an osmotic control for $\mathrm{HG}$, for 18 hours. Cells were incubated with either ${ }^{3} \mathrm{H}$-thymidine or ${ }^{3} \mathrm{H}$-leucin (1 
$\mu \mathrm{ci} / \mathrm{ml}$; Amersham Pharmacia Biotech) for 6 hours and the incorporation was counted by liquid scintillation counter (Hoshi et al, 2000).

\section{Western Blotting}

Glomeruli of 5-month-old ZDF-fa/fa and lean rats (three in each group) were isolated by the standard sieving method. The glomeruli or cultured podocytes were rinsed with PBS(-) and lysed with ice-cold buffer containing $0.1 \%$ TritonX-100, $0.4 \mathrm{M} \mathrm{NaCl}, 20 \mathrm{~mm}$ Tris-HCl, 1 mм EDTA, 1 mм EGTA, 1 mм DTT, 1 mм PMSF, $1 \mu \mathrm{g} / \mathrm{ml}$ aprotinin, $1 \mu \mathrm{g} / \mathrm{ml}$ leupeptin, $1 \mu \mathrm{g} / \mathrm{ml}$ antipain, $1 \mu \mathrm{g} / \mathrm{ml}$ pepstatinA, and $1 \mu \mathrm{g} / \mathrm{ml}$ chymostatin, and homogenized with 50 strokes in a Dounce homogenizer at $4^{\circ} \mathrm{C}$. Then, samples were centrifuged at $10,000 \mathrm{~g}$ for 5 minutes and supernatants were measured by protein assay (Bio-Rad, Hercules, California). Aliquots containing $40 \mu \mathrm{g}$ of protein from the glomerular extracts or $10 \mu \mathrm{g}$ of protein from the podocyte extracts were subjected to electrophoresis on $10 \%$ to $20 \%$ SDS gels. Protein was transferred onto polyvinylidene fluoride microporous membranes (Immobilon-P; Millipore Corporation, Bedford, Massachusetts). The membranes were incubated with antip21cip1(Santa Cruz), anti-p27kip1 (Transduction Laboratories, Lexington, Kentucky), anti-p57kip2 (Santa Cruz), or anti $\beta$-actin antibody (Sigma), and then horseradish peroxidase-linked donkey anti-mouse or anti-rabbit IgG antibody. Protein was visualized using an enhanced chemiluminescence kit (ECL Western blotting detection reagents; Amersham Pharmacia Biotech). Blots were scanned with an Image Master-CL (Amersham Pharmacia Biotech) and analyzed with NIH Image 1.55. Signal intensities in control lanes were arbitrarily assigned a value of 1.00 . Western blotting was independently repeated five times with qualitatively similar results.

\section{Statistical Analysis}

ANOVA was used to assess the significance of differences between groups. The paired $t$ test was used to assess the significance of difference within each group. The correlation coefficient was analyzed by Spearman rank correlation. Data are given as means \pm SEM. Statistical significance was assumed at $p<$ $0.05\left(^{*}\right)$ and $p<0.01\left(^{(*)}\right.$.

\section{Acknowledgements}

The authors thank Dr. Peter Mundel for providing the conditionally immortalized podocyte cell line. Critical discussion and reading of this manuscript by Dr. Kevin V. Lemley is gratefully appreciated.

\section{References}

Chomczynski P and Sacchi N (1998). Single-step method of RNA isolation by acid guanidinium thiocyanate-phenolchloroform extraction. Anal Biochem 162:156-159.

Cohen MP, Sharma K, Jin Y, Hud E, Wu Van-yu, Tamaszewski J, and Ziyadeh FN (1995). Prevention of diabetic ne- phropathy in $\mathrm{db} / \mathrm{db}$ mice with glycated albumin antagonists. $\mathrm{J}$ Clin Invest 95:2338-2345.

Coimbra TM, Janssen U, Groene JH, Ostendorf T, Kunter U, Schmidt H, Brabant G, and Floege J (2000). Early events leading to renal injury in obese zucker (fatty) rats with type II diabetes. Kidney Int 57:167-182.

Davidson WD and Sackner MA (1963). Simplification of the anthrone method for the determination of inulin in clearance studies. J Lab Clin Med 62:351-356.

Fiedorek FT (1996). Rodent genetic models for obesity and non-insulin-dependent diabetes mellitus. In: LeRoith D, Tayloe SI, and Olefsky JM, editors. Diabetes mellitus. Philadelphia: Lippincott-Raven, 604-618.

Godbole V and York DA (1978). Lipogenesis in situ in the genetically obese zucker fatty rat (fa/fa): Role of hyperphagia and hyperinsulinaemia. Diabetologia 14:191-197.

Goto Y, Kakizaki M, and Masaki N (1976). Production of spontaneous diabetic rats by repetition of selective breeding. Tohoku J Exp Med 119:85-90.

Herberg L and Coleman DL (1977). Laboratory animal exhibiting obesity and diabetes syndromes. Metabolism 26:59-99.

Hoshi S, Goto M, Koyama N, Nomoto K, and Tanaka H (2000). Regulation of vascular smooth muscle cell proliferation by nuclear factor-kappaB and its inhibitor, I-kappaB. J Biol Chem 275:883-889.

Hummel KP, Dickie MM, and Coleman DL (1996). Diabetes, new mutation in the mouse. Science 153:112-113.

Ikeda H, Shino A, Iwatsuka H, and Suzuoki Z (1976). A new genetically obese-hypergkycemic rat (Wistar fatty). Diabetes 30:1045-1050.

Kawano K, Hirashima T, and Mori S (1972). Spontaneous long-term hyperglycemic rat with diabetic complications: Otuka Long-Evans Tokushima Fatty (OLETF) strain. Diabetes 41:1422-1428.

Kriz W, Elger M, Nagata M, Kretzler M, Uiker S, KoeppenHageman I, Tenchert S, and Lemley K (1994). The role of podocytes in the development of glomerular sclerosis. Kidney Int 45:S-64-S-72.

Kriz W, Hahnel B, Rosener S, and Elger M (1995). Long-term treatment of rats with FGF-2 results in focal segmental glomerulosclerosis. Kidney Int 48:1435-1450.

Kuan CJ, al-Douahji M, and Shankland SJ (1998). The cyclin kinase inhibitor p21WAF1, CIP1 is increased in experimental diabetic nephropathy: Potential role in glomerular hypertrophy. J Am Soc Nephrol 9:986-993.

Mundel P, Reiser J, Zuniga Mejia Borja A, Pavenstadt H, Davidson GR, Kriz W, and Zeller R (1997). Rearrangements of the cytoskeleton and cell contacts induce process formation during differentiation of conditionally immortalized mouse podocytes cell lines. Exp Cell Res 236:248-258.

Nagata M and Kriz W (1992). Glomerular damage after uninephrectomy in young rats. II. Mechanical stress on podocytes as a pathway to sclerosis. Kidney Int 42:148-160.

Nagata M, Nakayama K, Terada Y, Hoshi S, and Watanabe T (1998). Cell cycle regulation and differentiation of the human podocytes lineage. Am J Pathol 153:1511-1520.

Nakamura M and Yamada K (1967). Studies on a diabetic strain of the mouse. Diabetologia 3:212-221. 
Nakamura S, Makita Z, Ishikawa S, Yasumura K, Fujii W, Yanagisawa K, Kawata T, and Koike T (1997). Progression of nephropathy in spontaneous diabetic rats is prevented by OPB-9195, a novel inhibitor of advanced glycation. Diabetes 46:895-899.

Nakamura T, Ushiyama C, Suzuki S, Hara M, Shimada N, Ebihara I, and Koide H (2000). Urinary excretion of podocytes in patients with diabetic nephropathy. Nephrol Dial Transplant 15:1379-1383.

Nelson RG, Meyer TW, Myers BD, and Bennett PH (1997). Clinical and pathological course of renal disease in noninsulin-dependent diabetes mellitus: The Pima Indian experience. Semin Nephrol 17:124-131.

Nitta K, Horita S, Honda K, Uchida K, Watanabe T, Nihei H, and Nagata M (1999). Glomerular expression of cell-cycleregulatory proteins in human crescentic glomerulonephritis. Virchows Arch 435:422-427.

Ophascharoensuk V, Fero ML, Hughes J, Roberts JM, and Shankland SJ (1998). The cyclin-dependent kinase inhibitor p27Kip1 safeguards against inflammatory injury. Nat Med 4:575-80.

Pagtalumn ME, Miller PL, Jumping-Eagles S, Nelson RG, Myers BD, Rennke HG, Coplon NS, Sun L, and Meyer TW (1997). Podocytes loss and progressive glomerular injury in type II diabetes. J Clin Invest 15:342-348.

Phillips AO, Janssen U, and Floege J (1999). Progression of diabetic nephropathy: Insights from cell culture studies and animal models. Kidney Blood Press Res 22:81-97.

Ritz E and Stefanski A (1996). Diabetic nephropathy in type II diabetes. Am J Kidney Dis 27:167-194.
Shankland ST, Floege J, Thomas SE, Nangaku M, Hugo C, Pippin J, Hennke K, Hockenberry DM, Johnson RJ, and Couser WG (1997). Cyclin kinase inhibitors are increased during experimental membranous nephropathy: Potential role in limiting glomerular epithelial cell proliferation in vivo. Kidney Int 52:404-413.

Tsuchida K, Makita Z, Yamagishi S, Atsumi T, Miyoshi H, Obara S, Ishida M, Ishikawa S, Yasumura K, and Koike T (1999). Suppression of transforming growth factor beta and vascular endothelial growth factor in diabetic nephropathy in rats by a novel advanced glycation end product inhibitor, OPB-91485. Diabetologia 42:579-588.

Vora JP, Zimsen SM, Houghton DC, and Anderson S (1996). Evolution of metabolic and renal changes in the ZDF/Drt-fa rat model of type II diabetes. J Am Soc Nephrol 7:113-117.

Wolf G, Schroender R, Thaiss F, Ziyadeh FN, Helmchen U, and Stahl RAK (1998). Glomerular expression of p27Kip1 in diabetic $\mathrm{db} / \mathrm{db}$ mouse: Role of hyperglycemia. Kidney Int 53:869-879.

Yagi K, Kim S, Wanibuchi H, Yamashita T, Yamanura Y, and Iwao H (1976). Characteristics of diabetes, blood pressure, and cardiac and renal complications in otsuka long-evans tokushima fatty rats. Hypertension 29:728-735.

Yaoita E, Kawasaki K, Yamamoto T, and Kihara I (1990). Variable expression of desmin in rat glomerular epithelial cells. Am J Pathol 136:899-908. 